\title{
Finger Assignment Schemes for RAKE Receivers with Multi-way Soft Handover*
}

\author{
Seyeong Choi \\ Dept. of Electrical \& Computer Eng. \\ Texas A\&M University \\ College Station, TX 77843, USA \\ Email: yeong@ece.tamu.edu
}

\author{
Mohamed-Slim Alouini, Khalid A. Qaraqe \\ Dept. of Electrical Eng. \\ Texas A\&M University at Qatar \\ Education City, Doha, Qatar \\ Email: \{alouini, khalid.qaraqe\}@qatar.tamu.edu
}

\author{
Hong-Chuan Yang \\ Dept. of Electrical \& Computer Eng. \\ University of Victoria \\ BC, V8W 3P6, Canada \\ Email: hyang@ece.uvic.ca
}

\begin{abstract}
We propose and analyze new finger assignment techniques that are applicable for RAKE receivers in the soft handover (SHO) region. More specifically, in the SHO region, the receiver uses by default only the strongest paths from the serving base station (BS) and only when the combined signal-to-noise ratio (SNR) falls below a certain pre-determined threshold, the receiver uses more resolvable paths from the target BSs to improve the performance. Relying on the previous results for the case of two-BS case, we consider the multi-BS situation by attacking the statistics of several correlated generalized selection combining (GSC) stages and provide closed-form expressions for the statistics of the output SNR. By investigating the tradeoff among the error performance, the path estimation load, and the SHO overhead, we show through numerical examples that the new schemes offer commensurate performance in comparison with more complicated GSC-based diversity systems while requiring a smaller estimation load and SHO overhead.
\end{abstract}

Keywords - Fading channels, diversity techniques, RAKE receiver, generalized selection combining (GSC), performance analysis.

\section{INTRODUCTION}

RAKE reception is a technique which uses several baseband correlators called fingers to individually process multi-path signal components. The outputs from the different correlators are coherently combined to improve the signal-to-noise ratio (SNR) and to therefore lower the probability of deep fades [1, Section 9.5.1]. Since they rely on resolvable multi-paths to operate, RAKE receivers are used in conjunction with wideband systems such as wideband code division multiple access (WCDMA) and ultra wideband (UWB) systems. Due to the hardware and battery life time constraints, we now resort to finding solutions for the better combining schemes that achieve low complexity and low power consumption while offering a minimal usage of additional network resources.

Although many low-complexity diversity combining schemes have been proposed and studied over the last decade [2]-[12], they still result in a significant increase in network overhead (known as a soft handover (SHO) overhead) if they are used without any modification. Recently, the authors proposed and analyzed the performance of a new finger assignment scheme that maintains a low complexity and reduces the SHO overhead [13], [14]. The main idea

* This work was supported in part by the Qatar Foundation for Education, Science, and Community Development, Qatar, in part by Qatar Telecom (Qtel), Qatar, and in part by a Discovery Grant from NSERC, Canada. behind [14] is that, in the SHO region whenever the received signal is unsatisfactory, the receiver scans the additional resolvable paths from the target base station (BS) and selects the strongest paths among the total available paths from both the serving and the target BS. However, in [14] only two BSs are assumed (one serving and one target BSs). In this paper, we generalize the results of [14] to the multi-BS situation. We propose two assignment schemes denoted as the full scanning scheme and the sequential scanning scheme. For the full scanning scheme, whenever the generalized selection combining (GSC) output SNR of the paths from the serving $\mathrm{BS}$ is below a certain pre-determined threshold (known as the target SNR), the RAKE receiver scans all the available paths from all the target BSs while for the sequential scanning scheme, the RAKE receiver sequentially scans the target BSs until the combined SNR is satisfactory or all target BSs are scanned.

The main contribution of this paper is to provide an analytical framework deriving the statistics of the receiver output SNR of our proposed schemes, including the cumulative density function (CDF), probability density function (PDF), and moment generating function (MGF) of the output SNR. In our derivations, we specifically tackle the statistics of the output SNR which is the sum of correlated GSC output. These results are then used first to analyze the performance in terms of the average probability of error and then to investigate the tradeoff between complexity and performance by quantifying the average number of path estimations and the SHO overhead versus the target SNR. To simplify our analysis and make it tractable, we assume that the receiver operates over a "perfect" uniform propagation delay profile provided by a multi-path searcher in a way that the multi-path components are correctly assigned to the RAKE fingers.

The remaining of this paper is organized as follows. In Section II, we present the system and channel model under consideration as well as the mode of operation of the proposed schemes. Based on this mode of operation, we derive the expressions for the statistics of the combined SNR in Section III. These results are next applied to the performance analysis of the proposed systems in Section IV. This section also illustrates the tradeoff of complexity versus performance by comparing the number of path estimations and the SHO overhead of our proposed systems to that of conventional GSC and maximal ratio combining (MRC). Finally, Section V provides some concluding remarks. 


\section{System Model}

\section{A. Channel and System Model}

Let $\gamma_{j}$ denote the instantaneous received SNR of the $j$ th resolvable path, $j=1,2, \cdots, \sum_{i=1}^{N} L_{i}$, where $L_{i}$ is the number of resolvable paths from $i$ th $\mathrm{BS}$ and $N$ is the number of available BSs in the SHO region. We assume that the signals from all the resolvable paths experience independent and identically distributed (i.i.d.) Rayleigh fading environments ${ }^{1}$. Under a block fading assumption, the fading channel gain of each path is assumed to be constant over one time slot and vary independently from one slot to the next. As such, the faded SNR, $\gamma_{j}$, follows the same exponential distribution with the common average faded SNR, $\bar{\gamma}$.

Next, we consider systems that employ a RAKE receiver with GSC. We assume that the RAKE receiver has $L_{c}$ fingers and, in the SHO region, depending on the channel conditions only $L_{c}$ paths among $L_{(k)}$ paths are used for RAKE reception where $L_{(k)}=\sum_{i=1}^{k} L_{i}$ and $1 \leq k \leq N$. Now if we let $\Gamma_{a: b}$ be the sum of the $a$ largest SNRs among $b$ ones, i.e., $\Gamma_{a: b}=\sum_{d=1}^{a} \gamma_{d: b}$ where $\gamma_{d: b}$ is the $d$ th order statistics (see [4] for terminology), then the total received SNR after GSC is given by $\Gamma_{L_{c}: L_{(k)}}$.

\section{B. Mode of Operation}

For convenience, let $L_{1}$ be the number of resolvable paths from the serving $\mathrm{BS}$ and $L_{2}, L_{3}, \cdots, L_{N}$ be those from the target BSs. Without loss of generality, we assume that at first the receiver relies only on $L_{1}$ resolvable paths and as such starts with $L_{c} / L_{1}$-GSC. In the SHO region, the receiver compares the received SNR, $\Gamma_{L_{c}: L_{1}}$, with a certain target SNR, denoted by $\gamma_{T}$. If $\Gamma_{L_{c}: L_{1}}$ is greater than or equal to $\gamma_{T}$, a one-way $\mathrm{SHO}^{2}$ is used and no finger reassignment is needed. On the other hand, whenever $\Gamma_{L_{c}: L_{1}}$ falls below $\gamma_{T}$, a multiway $\mathrm{SHO}^{3}$ is attempted. More specifically, we consider two different finger assignment schemes described below.

1) Case I - Full Scanning: In this case, when $\Gamma_{L_{c}: L_{1}}<$ $\gamma_{T}$, the RAKE at once scans all possible $L_{(N)}$ resolvable paths from $N$ BSs and reassigns its $L_{c}$ fingers to the $L_{c}$ strongest paths among the $L_{(N)}$ available resolvable paths (i.e., the RAKE receiver uses $L_{c} / L_{(N)}$-GSC). Hence, the final combined SNR, denoted by $\gamma_{F u l l}$, is mathematically given by

$$
\gamma_{\text {Full }}= \begin{cases}\Gamma_{L_{c}: L_{1}}, & \gamma_{T} \leq \Gamma_{L_{c}: L_{1}} \\ \Gamma_{L_{c}: L_{(N)}}, & \Gamma_{L_{c}: L_{1}}<\gamma_{T}\end{cases}
$$

2) Case II - Sequential Scanning: In this case, when $\Gamma_{L_{c}: L_{1}}<\gamma_{T}$, the RAKE receiver estimates $L_{2}$ paths from the first target $\mathrm{BS}$ and uses $L_{c} / L_{(2)}$-GSC. The receiver then checks whether the combined SNR, $\Gamma_{L_{c}: L_{(2)}}$, is above $\gamma_{T}$ or

\footnotetext{
${ }^{1}$ In [15], more practical channel environments, such as nonidentical/correlated fading channels and outdated channel estimation, are considered.

${ }^{2}$ One-way SHO refers to the scenario in which the mobile unit is connected only to the serving BS while being in the SHO region.

${ }^{3}$ Multi-way SHO refers to the scenario in which the mobile unit is connected to the serving BS and the target BSs while being in the SHO region.
}

not. By sequentially adding the remaining target BSs, this process is repeated until either the combined SNR, $\Gamma_{L_{c}: L_{(k)}}$, is above $\gamma_{T}$ or all the $L_{(N)}$ paths are examined. Based on this mode of operation, we can see that the final combined SNR, denoted by $\gamma_{S e q}$, is mathematically given by

$$
\gamma_{S e q}=\left\{\begin{array}{cl}
\Gamma_{L_{c}: L_{1}}, & \gamma_{T} \leq \Gamma_{L_{c}: L_{1}} ; \\
\Gamma_{L_{c}: L_{(2)}}, & \Gamma_{L_{c}: L_{1}}<\gamma_{T} \leq \Gamma_{L_{c}: L_{(2)}} \\
\vdots & \vdots \\
\Gamma_{L_{c}: L_{(N-1)},} & \Gamma_{L_{c}: L_{(N-2)}}<\gamma_{T} \leq \Gamma_{L_{c}: L_{(N-1)}} \\
\Gamma_{L_{c}: L_{(N)}}, & \Gamma_{L_{c}: L_{(N-1)}}<\gamma_{T} .
\end{array}\right.
$$

\section{Statistics OF COMBINED SNR}

Although the mode of operations in (1) and (2) describe a scheme that essentially switches among $L_{c} / L_{(k)}$-GSC stages depending on the channel conditions and the output threshold, we can not obtain the statistics of $\gamma_{F u l l}$ and $\gamma_{S e q}$ directly from that of the output SNR with conventional GSC. Hence, in this section, we rely on some recently derived order statistics results [14] to derive the statistics of the combined SNRs of $\gamma_{F u l l}$ and $\gamma_{S e q}$.

\section{A. Case I - Full Scanning}

Comparing (1) and [14, Eq. (3)], we can see that the results in [14] can be directly used. The key difference is that $L$ and $L+L_{a}$ in [14] have to be replaced here by $L_{1}$ and $L_{(N)}$, respectively.

\section{B. Case II - Sequential Scanning}

If we let $L_{t}$ be the number of total resolvable paths examined for the finger assignment, then applying the total probability theorem, we can write the CDF of combined SNR, $\gamma_{S e q}$, as

$$
\begin{aligned}
F_{\gamma_{S e q}}(x) & =\operatorname{Pr}\left[\gamma_{S e q}<x\right] \\
& =\sum_{k=1}^{N} \operatorname{Pr}\left[\gamma_{S e q}<x, L_{t}=L_{(k)}\right]
\end{aligned}
$$

Note that based on the mode of operation, $L_{(k)}(k<N)$ paths are examined if and only if the GSC-combined SNR of the first $L_{(k-1)}$ paths is less than $\gamma_{T}$ but the combined SNR of the first $L_{(k)}$ paths is greater than or equal to $\gamma_{T}$. In addition, if the combined SNR of $L_{(N-1)}$ paths is below $\gamma_{T}$, then $L_{c} / L_{(N)^{-}}$ GSC is used. Hence, the joint probability in (3) can be written as

$$
\begin{aligned}
& \operatorname{Pr}\left[\gamma_{S e q}<x, L_{t}=L_{(k)}\right] \\
& =\left\{\begin{array}{cl}
\operatorname{Pr}\left[\gamma_{T} \leq \Gamma_{L_{c}: L_{1}}<x\right], \quad k=1 ; \\
\operatorname{Pr}\left[\gamma_{T} \leq \Gamma_{L_{c}: L_{(k)}}<x,\right. \\
\Gamma_{\left.L_{c}: L_{(k-1)}<\gamma_{T}\right],}<\leq k \leq N-1 ; \\
\operatorname{Pr}\left[\Gamma_{L_{c}: L_{(N)}<x,}<\gamma_{T}\right], \quad k=N .
\end{array}\right.
\end{aligned}
$$


Substituting (4) into (3), we can obtain the CDF of $\gamma_{S e q}$ as

$$
\begin{aligned}
F_{\gamma_{S e q}}(x) & =\operatorname{Pr}\left[\gamma_{T} \leq \Gamma_{L_{c}: L_{1}}<x\right] \\
& +\sum_{k=2}^{N-1} \operatorname{Pr}\left[\gamma_{T} \leq \Gamma_{L_{c}: L_{(k)}}<x, \Gamma_{L_{c}: L_{(k-1)}}<\gamma_{T}\right] \\
& +\operatorname{Pr}\left[\Gamma_{L_{c}: L_{(N)}}<x, \Gamma_{L_{c}: L_{(N-1)}}<\gamma_{T}\right] .
\end{aligned}
$$

Since it is clear that $\Gamma_{L_{c}: L_{(N-1)}} \leq \Gamma_{L_{c}: L_{(N)}}$, we can rewrite $\operatorname{Pr}\left[\Gamma_{L_{c}: L_{(N)}}<x, \Gamma_{L_{c}: L_{(N-1)}}<\gamma_{T}\right]$ in (5) as

$$
\begin{aligned}
& \operatorname{Pr}\left[\Gamma_{L_{c}: L_{(N)}}<x, \Gamma_{L_{c}: L_{(N-1)}}<\gamma_{T}\right] \\
& = \begin{cases}\operatorname{Pr}\left[\Gamma_{L_{c}: L_{(N)}}<x\right], & 0 \leq x<\gamma_{T} ; \\
\operatorname{Pr}\left[\Gamma_{L_{c}: L_{(N)}}<\gamma_{T}\right] & \\
+\operatorname{Pr}\left[\gamma_{T} \leq \Gamma_{L_{c}: L_{(N)}}<x,\right. & \\
\left.\Gamma_{L_{c}: L_{(N-1)}}<\gamma_{T}\right], & x \geq \gamma_{T} .\end{cases}
\end{aligned}
$$

Substituting (6) into (5) and using the derivation in [14, Appendix], we can obtain the CDF and the PDF of $\gamma_{S e q}$ as

$$
\begin{aligned}
& F_{\gamma_{S e q}}(x) \\
& =\left\{\begin{array}{l}
\operatorname{Pr}\left[\Gamma_{L_{c}: L_{(N)}}<x\right], \\
\operatorname{Pr}\left[\gamma_{T} \leq \Gamma_{L_{c}: L_{1}}<x\right] \\
+\operatorname{Pr}\left[\Gamma_{L_{c}: L_{(N)}}<\gamma_{T}\right] \\
+\sum_{k=2}^{N}\left\{\operatorname{Pr}\left[\gamma_{T} \leq \Gamma_{L_{c}: L_{(k)}}<x\right]\right. \\
-\frac{1-\operatorname{Pr}\left[\Gamma_{L_{c}: L_{(k-1)}}<\gamma_{T}\right]}{1-\operatorname{Pr}\left[\Gamma_{\left.L_{c}: L_{(k)}-1<\gamma_{T}\right]}\right.} \\
\left.\times\left(\operatorname{Pr}\left[\gamma_{T} \leq \Gamma_{L_{c}: L_{(k)}}<x\right]-\mathcal{J}(x)\right)\right\}, x \geq \gamma_{T} ;
\end{array}\right.
\end{aligned}
$$

and

$$
f_{\gamma_{S e q}}(x)= \begin{cases}f_{\Gamma_{L_{c}: L_{(N)}}}(x), & 0 \leq x<\gamma_{T} ; \\ f_{\Gamma_{L_{c}: L_{1}}}(x) & \\ +\sum_{k=2}^{N}\left[f_{\Gamma_{L_{c}: L_{(k)}}}(x)\right. & \\ -\frac{1-F_{\Gamma_{L_{c}: L_{(k-1)}}}\left(\gamma_{T}\right)}{1-F_{\Gamma_{L_{c}: L_{(k)}}-1}\left(\gamma_{T}\right)} & \\ \left.\times\left(f_{\Gamma_{L_{c}: L_{(k)}}}(x)-\mathcal{I}(x)\right)\right], & x \geq \gamma_{T},\end{cases}
$$

respectively, where

$$
\begin{aligned}
\mathcal{I}(x) & =\frac{d}{d x} \mathcal{J}(x) \\
& =\frac{d}{d x} \operatorname{Pr}\left[\gamma_{T} \leq \Gamma_{L_{c}: L_{(k)}}<x, \Gamma_{L_{c}: L_{(k)}-1}<\gamma_{T}\right] .
\end{aligned}
$$

Note that for i.i.d. Rayleigh fading channels, $f_{\Gamma_{i: j}}(x)$ and $F_{\Gamma_{i: j}}(x)$ are the well-known PDF and CDF of $i / j$-GSC output SNR which can be found in [16, Eqs. (9.433)(9.440)], respectively, and (9) can be obtained by using the result in [14, Eq. (18)]. Therefore, (7) and (8) can be expressed in closedform. With the PDF of (8) in hand, the closed-form expression for MGF of $\gamma_{S e q}, \mathcal{M}_{\gamma_{S e q}}(s)=\int_{0}^{\infty} e^{s x} f_{\gamma_{S e q}}(x) d x$, can be routinely obtained after lengthy and tedious calculations.

\section{Performance Analysis}

In this section, we apply the closed-form results of the previous section to analyze the performance of our proposed combining scheme over Rayleigh fading channels. More specifically, we first examine its average bit error rate (BER) by using the well-known MGF-based approach [16, Sec. 9.2.3]. We then look into the average number of path estimations and the SHO overhead it requires.

\section{A. Average BER Comparison}

Fig. 1 represents the average BER of binary phase shift keying (BPSK) versus the average SNR per branch, $\bar{\gamma}$, of the proposed schemes for various values of $\gamma_{T}$ over i.i.d. Rayleigh fading channels when $N=4, L_{1}=L_{2}=L_{3}=L_{4}=4$, and $L_{c}=3$. For comparison purpose, we also plot the average BER of BPSK with $L_{c}$-MRC, $L_{c} / L_{1}$-GSC, and $L_{c} / L_{(N)^{-}}$ GSC. From this figure, it is clear that the higher the threshold, the better performance, as one expects. As a check, we can see that when the threshold is too large (i.e., $\gamma_{T}=15 \mathrm{~dB}$ ) or too small (i.e., $\gamma_{T}=-5 \mathrm{~dB}$ ), both schemes have almost the same performance which correspond to the performance of $L_{c} / L_{(N)}$-GSC for the high threshold and $L_{c} / L_{1}$-GSC for the low threshold. For the mid-range of the output threshold (i.e., $\gamma_{T}=5 \mathrm{~dB}$ ), the full scanning scheme has slightly better performance than the sequential scheme. However, with this slight (negligible) performance loss, the sequential scheme can save the usage of the network resources compared to the full scanning scheme since it can dramatically reduce the unnecessary path estimations and the SHO overhead, as we show in what follows.

\section{B. Average Number of Path Estimations}

1) Case I - Full Scanning: With this case, the RAKE receiver estimates $L_{1}$ paths in the case of $\Gamma_{L_{c}: L_{1}} \geq \gamma_{T}$ or $L_{(N)}$ in the case of $\Gamma_{L_{c}: L_{1}}<\gamma_{T}$. Hence, we can easily quantify the average number of path estimations, denoted by $N_{F u l l}$, as

$$
\begin{aligned}
& N_{\text {Full }} \\
& =L_{1} \operatorname{Pr}\left[\Gamma_{L_{c}: L_{1}} \geq \gamma_{T}\right]+L_{(N)} \operatorname{Pr}\left[\Gamma_{L_{c}: L_{1}}<\gamma_{T}\right],
\end{aligned}
$$

which reduces to

$$
N_{F u l l}=L_{1}+\left(L_{(N)}-L_{1}\right) F_{\Gamma_{L_{c}: L_{1}}}\left(\gamma_{T}\right) .
$$

2) Case II - Sequential Scanning: In this scheme, we can write the average number of path estimations, denoted by $N_{\text {Seq }}$, in the following summation form:

$$
N_{S e q}=\sum_{l=1}^{N} L_{(l)} \cdot \pi_{l},
$$

where $\pi_{l}$ is the probability that $L_{(l)}$ paths are estimated. Based on the mode of operation, we have

$$
\pi_{l}= \begin{cases}\operatorname{Pr}\left[\Gamma_{L_{c}: L_{1}} \geq \gamma_{T}\right], & l=1 ; \\ \operatorname{Pr}\left[\Gamma_{L_{c}: L_{(l-1)}}<\gamma_{T}, \Gamma_{L_{c}: L_{(l)}} \geq \gamma_{T}\right], & 1<l<N ; \\ \operatorname{Pr}\left[\Gamma_{L_{c}: L_{(N-1)}}<\gamma_{T}\right], & l=N .\end{cases}
$$


By the similar approach used in order to get (7), the joint probability in (13) can be obtained as

$$
\begin{aligned}
& \operatorname{Pr}\left[\Gamma_{L_{c}: L_{(l-1)}}<\gamma_{T}, \Gamma_{L_{c}: L_{(l)}} \geq \gamma_{T}\right] \\
& =\operatorname{Pr}\left[\gamma_{T} \leq \Gamma_{L_{c}: L_{(l)}}\right]-\frac{1-\operatorname{Pr}\left[\Gamma_{L_{c}: L_{(l-1)}}<\gamma_{T}\right]}{1-\operatorname{Pr}\left[\Gamma_{L_{c}: L_{(l)}-1}<\gamma_{T}\right]} \\
& \quad \times\left(\operatorname{Pr}\left[\gamma_{T} \leq \Gamma_{L_{c}: L_{(l)}}\right]-\mathcal{K}(l)\right)
\end{aligned}
$$

where

$$
\begin{aligned}
& \mathcal{K}(l)=\operatorname{Pr}\left[\Gamma_{L_{c}: L_{(l)}-1}<\gamma_{T}, \Gamma_{L_{c}: L_{(l)}} \geq \gamma_{T}\right] \\
& =e^{-\frac{\gamma_{T}}{\bar{\gamma}}}\left(\frac{\gamma_{T}}{\bar{\gamma}}\right)^{L_{c} L_{(l)}-L_{c}-1} \sum_{t=0}^{L_{c}-1} \sum_{u=0} \\
& \frac{(-1)^{t+u}\left(\begin{array}{c}
L_{(l)}-1 \\
L_{c}, L_{(l)}-L_{c}-t-1, t
\end{array}\right)}{\left(L_{c}-u-1\right) !\left((t+1) \gamma_{T} /\left(\bar{\gamma} L_{c}\right)\right)^{u+1}} \\
& \times\left[1-e^{-\frac{(t+1) \gamma_{T}}{\bar{\gamma} L_{c}}} \sum_{v=0}^{u}\left(\frac{(t+1) \gamma_{T}}{\bar{\gamma} L_{c}}\right)^{v} / v !\right] .
\end{aligned}
$$

After successive substitutions from (15) to (12), we can express the average number of path estimations, $N_{S e q}$, as

$$
\begin{aligned}
& N_{S e q} \\
& =L_{(1)}\left(1-F_{\Gamma_{L_{c}: L_{1}}}\left(\gamma_{T}\right)\right)+L_{(N)} F_{\Gamma_{L_{c}: L_{(N-1)}}}\left(\gamma_{T}\right) \\
& \quad+\sum_{l=2}^{N-1} L_{(l)}\left(1-F_{\Gamma_{L_{c}: L_{(l)}}}\left(\gamma_{T}\right)-\frac{1-F_{\Gamma_{L_{c}: L_{(}(l-1)}}\left(\gamma_{T}\right)}{1-F_{\Gamma_{L_{c}: L_{(}(l)}}\left(\gamma_{T}\right)}\right. \\
& \left.\quad \times\left(1-F_{\Gamma_{L_{c}: L_{(l)}}}\left(\gamma_{T}\right)-\mathcal{K}(l)\right)\right) .
\end{aligned}
$$

Fig. 2 shows the average number of path estimations versus the output threshold, $\gamma_{T}$, of the proposed schemes, MRC, and GSC for various values of $L_{c}$ over i.i.d. Rayleigh fading channels when $N=4, L_{1}=L_{2}=L_{3}=L_{4}=4$, and $\bar{\gamma}=0$ $\mathrm{dB}$. Note that $L_{c}$-MRC and $L_{c} / L_{(N)}$-GSC always require $L_{c}$ and $L_{(N)}$ estimations, respectively. From this figure, we can clearly see that the sequential scanning scheme leads to a considerably less path estimation load. For a better illustration of the tradeoff between complexity and performance, Fig. 3 shows the average BER of BPSK versus the output threshold, $\gamma_{T}$, of the proposed schemes, MRC, and GSC for the same parameters. As mentioned earlier, the full scanning scheme shows a very slight performance improvement and the error rate of both proposed schemes decreases to that of $L_{c} / L_{(N)^{-}}$ GSC when the output threshold increases. Considering Figs. 2 and 3 together, we observe that the proposed schemes can save a certain amount of estimation load with a slight performance loss compared to GSC if the transmitted power is properly selected such as, for example, the average received SNR is $4 \sim 6 \mathrm{~dB}$ below the required target threshold for our chosen set of parameters.

\section{SHO Overhead}

The SHO overhead, denoted by $\beta$, is commonly used to quantify the SHO activity in a network and is defined as [17,
Eq. (9.2)]

$$
\beta=\sum_{n=1}^{L_{c}} n P_{n}-1,
$$

where $L_{c}(\leq N)$ is the number of fingers (i.e., the number of active BSs) and $P_{n}$ is the average probability that the mobile unit uses $n$-way SHO. Note that basically a maximum $L_{c}$-way SHO is possible and we need to determine which BSs the combined $L_{c}$ paths are from. Unfortunately, it seems difficult to analyze the SHO overhead in a simple fashion. The major difficulty lies in how to determine how many BSs end up eventually being involved in the SHO (i.e., the number $n$ in $n$-way $\mathrm{SHO}$ ) after $\mathrm{HO}$ is requested. For this reason, we just present some numerical results obtained through Monte-Carlo simulations.

In Fig. 4, we plot the simulation results of SHO overhead versus the output threshold, $\gamma_{T}$, of the full scanning and sequential scanning schemes for various values of $L_{c}$ over i.i.d. Rayleigh fading channels for the same parameters used in Figs. 2 and 3. Note that as the output threshold increases we have a higher chance to use $L_{c}$-way SHO. Compared to the full scanning scheme, for the mid-range of the output threshold the sequential scanning scheme shows a large amount of reduction of the SHO overhead. From this figure together with Fig. 3, we can quantify the SHO overhead reduction of our proposed schemes. For example, if the required threshold is $8 \mathrm{~dB}$ above $\bar{\gamma}$ in the case of $L_{c}=3$, the full scanning and the sequential scheme shows around $65 \%$ and $20 \%$ of the maximum SHO overhead, respectively, while maintaining the same error rate as GSC (which requires 100\% SHO overhead).

\section{CONClusion}

In this paper, we proposed new finger assignment schemes that are applicable for RAKE receivers operating in the SHO region. In these schemes, the receiver checks the GSC output SNR from the serving BS against a certain pre-determined output threshold. If the output SNR is below this threshold, the receiver performs a finger reassignment after using GSC on the paths coming from the serving BS and the target BSs. More specifically, we considered two schemes : a full scanning scheme and a sequential scanning scheme. For both schemes, we derived the statistics of the output SNR, based on which we carried out the performance analysis of the resulting systems. We showed through numerical examples that the new schemes offer commensurate performance in comparison with more complicated GSC-based diversity systems while requiring a smaller estimation load and SHO overhead.

\section{REFERENCES}

[1] G. L. Stüber, Principles of Mobile Communication, 2nd ed. Norwell, MA: Kluwer Academic Publishers, 2001.

[2] T. Eng, N. Kong, and L. B. Milstein, "Comparison of diversity combining techniques for Rayleigh-fading channels," IEEE Trans. Commun., vol. 44, no. 9, pp. 1117-1129, Sept. 1996.

[3] M. Z. Win and J. H. Winters, "Analysis of hybrid selection/maximalratio combining in Rayleigh fading," IEEE Trans. Commun., vol. 47, no. 12, pp. 1773-1776, Dec. 1999. 
[4] M.-S. Alouini and M. K. Simon, "An MGF-based performance analysis of generalized selection combining over Rayleigh fading channels," IEEE Trans. Commun., vol. 48, no. 3, pp. 401-415, Mar. 2000.

[5] Y. Ma and C. C. Chai, "Unified error probability analysis for generalized selection combining in Nakagami fading channels," IEEE J. Select. Areas Commun., vol. 18, no. 11, pp. 2198-2210, Nov. 2000.

[6] A. Annamalai and C. Tellambura, "Analysis of hybrid selection/maximal-ratio diversity combiner with Gaussian errors," IEEE Trans. Wireless Commun., vol. TWC-1, no. 3, pp. 498-512, July 2002.

[7] S. W. Kim, D. S. Ha, and J. H. Reed, "Minimum selection GSC and adaptive low-power RAKE combining scheme," in Proc. IEEE Int. Symp. on Circuit and Systems (ISCAS'03), Bangkok, Thailand, May 2003.

[8] H.-C. Yang, "Exact performance analysis of minimum-selection generalized selection combining (GSC)," in Proc. IEEE Int. Conf. on Commun. (ICC'05), Seoul, Korea, May 2005.

[9] - "New results on ordered statistics and analysis of minimumselection generalized selection combining (GSC)," IEEE Trans. Wireless Commun., vol. 5, no. 7, pp. 1876-1885, July 2006.

[10] R. K. Mallik, P. Gupta, and Q. T. Zhang, "Minimum selection GSC in independent Rayleigh fading," IEEE Trans. Veh. Technol., vol. 54, no. 3, pp. 1013-1021, May 2005 .

[11] M.-S. Alouini and H.-C. Yang, "Minimum estimation and combining generalized selection combining (MEC-GSC)," in Proc. IEEE Int. Symp. on Information Theory (ISIT'05), Adelaide, Australia, Sept. 2005.

[12] H.-C. Yang and M.-S. Alouini, "MRC and GSC diversity combining with an output theshold," IEEE Trans. Veh. Technol., vol. 54, no. 3, pp. 1081-1090, May 2005.

[13] S. Choi, M.-S. Alouini, K. A. Qaraqe, and H.-C. Yang, "Performance analysis of RAKE receivers with finger reassignment," in Proc. IEEE Int. Conf. on Commun. Systems (ICCS'06), Singapore, Nov. 2006.

[14] _ "Soft handover overhead reduction by RAKE reception with finger reassignment," IEEE Trans. Commun., under revision.

[15] _ - "Finger replacement schemes for RAKE receivers in the soft handover region with multiple base station," IEEE Trans. Wireless Commun., submitted.

[16] M. K. Simon and M.-S. Alouini, Digital Communication over Fading Channels, 2nd ed. New York, NY: John Wiley \& Sons, 2005.

[17] H. Holma and A. Toskala, WCDMA for UMTS, revised ed. New York, NY: John Wiley \& Sons, 2001

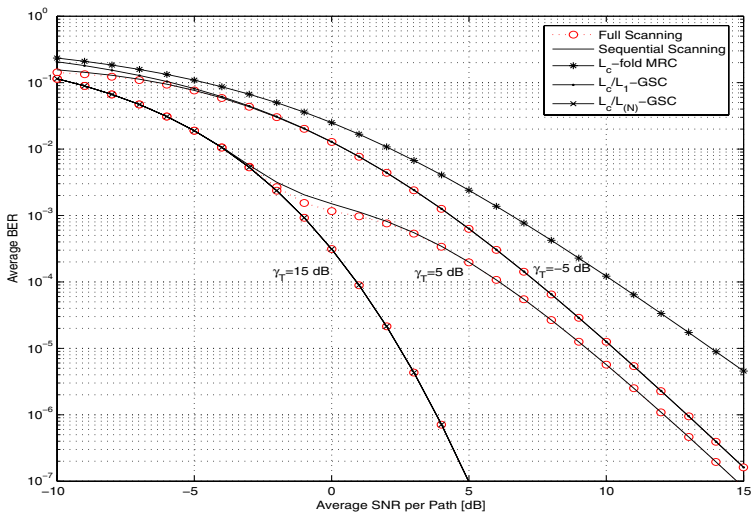

Fig. 1. Average BER of BPSK versus the average SNR per branch, $\bar{\gamma}$, of the full scanning and sequential scanning schemes, MRC, and GSC for various values of $\gamma_{T}$ over i.i.d. Rayleigh fading channels with $N=4, L_{1}=L_{2}=$ $L_{3}=L_{4}=4$, and $L_{c}=3$.

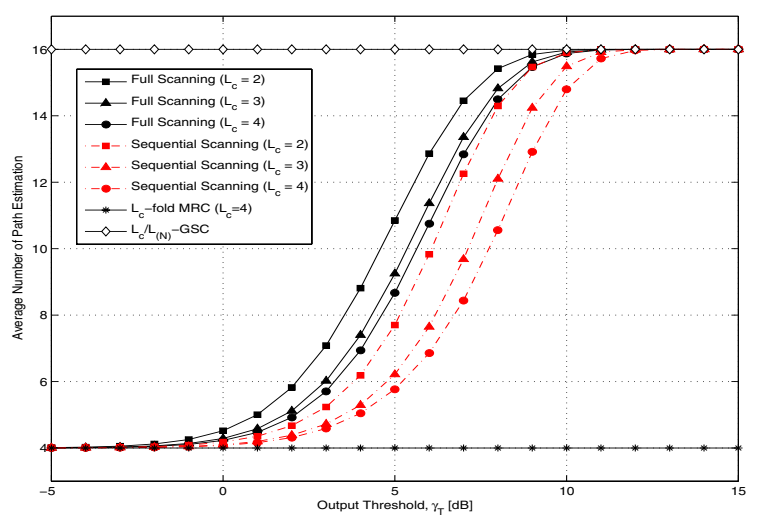

Fig. 2. Average number of path estimation versus the output threshold, $\gamma_{T}$, of the full scanning and sequential scanning schemes, MRC, and GSC for various values of $L_{c}$ over i.i.d. Rayleigh fading channels with $N=4, L_{1}=$ $L_{2}=L_{3}=L_{4}=4$, and $\bar{\gamma}=0 \mathrm{~dB}$

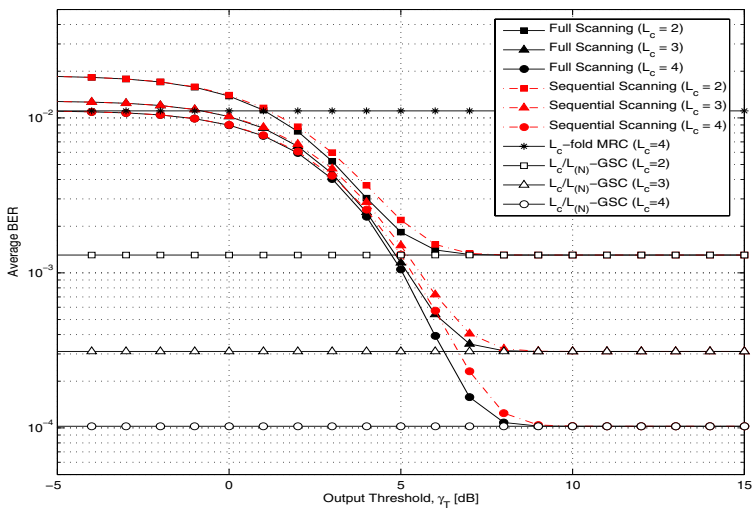

Fig. 3. Average BER of BPSK versus the output threshold, $\gamma_{T}$, of the full scanning and sequential scanning schemes, MRC, and GSC for various values of $L_{c}$ over i.i.d. Rayleigh fading channels with $N=4, L_{1}=L_{2}=L_{3}=$ $L_{4}=4$, and $\bar{\gamma}=0 \mathrm{~dB}$.

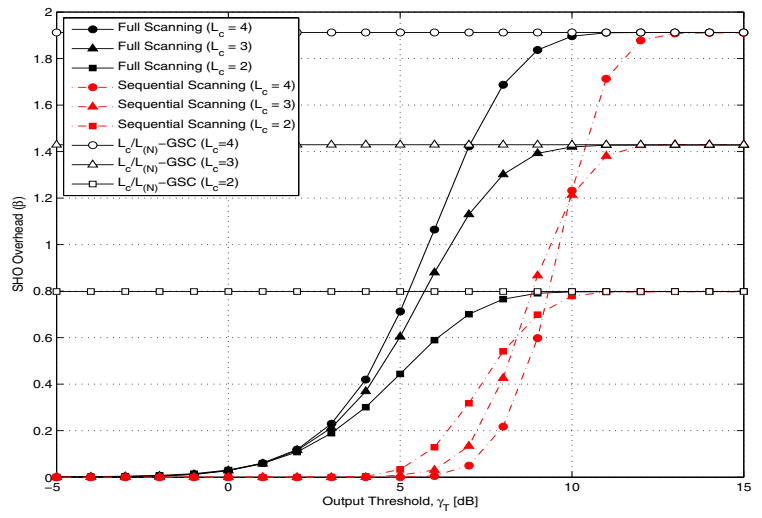

Fig. 4. Simulation results of SHO overhead versus the output threshold, $\gamma_{T}$, of the full scanning and sequential scanning schemes for various values of $L_{c}$ over i.i.d. Rayleigh fading channels with $N=4, L_{1}=L_{2}=L_{3}=L_{4}=4$, and $\bar{\gamma}=0 \mathrm{~dB}$. 